\title{
Relationship between damage and mortality in juvenile-onset systemic lupus erythematosus: Cluster analyses in a large cohort from the Spanish Society of Rheumatology Lupus Registry (RELESSER)
}

\section{Torrente-Segarra ${ }^{\mathrm{a}, *}$, T.C. Salman Monte ${ }^{\mathrm{b}}$, I. Rúa-Figueroa ${ }^{\mathrm{c}}$, J. De Uña-Álvarez ${ }^{\mathrm{d}}$, V. Balboa-Barreiro ${ }^{\mathrm{d}}$, F.J. López-Longo ${ }^{\mathrm{e}}$, M. Galindo-Izquierdo ${ }^{\mathrm{f}}$, J. Calvo-Alén ${ }^{\mathrm{g}}$,}

A. Olivé-Marqués ${ }^{h}$, C. Mouriño-Rodríguez ${ }^{i}$, L. Horcada $^{j}$, A. Sánchez-Atrio ${ }^{k}$, C. Montillal, E. Salgado ${ }^{\mathrm{m}}$, E. Díez-Álvarez $^{\mathrm{n}}$, R. Blanco ${ }^{\circ}$, J.L. Andreu ${ }^{\mathrm{p}}$, O. Fernández-Berrizbeitia ${ }^{\mathrm{q}}$, J.A. Hernández-Beriain ${ }^{r}$, M. Gantes ${ }^{\mathrm{s}}$, B. Hernández-Cruz ${ }^{\mathrm{t}}$, A. Pecondón-Español ${ }^{\mathrm{u}}, \mathrm{C}_{\text {. Marras }}^{\mathrm{v}}$, G. Bonillaw ${ }^{\mathrm{w}}$, J.M. Pego-Reigosa ${ }^{\mathrm{x}}$, On behalf of the RELESSER Study Group of the Spanish Society of Rheumatology (SER) and the Study Group of Systemic Autoimmune Diseases of the SER (EAS-SER)

\footnotetext{
a Rheumatology Department, Hospital General Hospitalet-Sant Joan DespíMoisèsBroggi, C/ Josep Molins 29-41, 08906 Hospitalet, Llobregat 08906, Spain

${ }^{\mathrm{b}}$ Rheumatology Department, Parc de Salut Mar-IMIM, Barcelona, Spain

' Rheumatology Department, Doctor Negrín University Hospital of Gran Canaria, Las Palmas de Gran Canaria, Spain

${ }^{\mathrm{d}}$ Department of Statistics and OR, Vigo University, Vigo, Spain

e Rheumatology Department, Gregorio Marañón University Hospital, Madrid, Spain

${ }^{\mathrm{f}}$ Rheumatology Department, Doce de Octubre University Hospital, Madrid, Spain

${ }^{\mathrm{g}}$ Rheumatology Department, Sierrallana Hospital, Torrelavega, Spain

${ }^{\mathrm{h}}$ Rheumatology Department, Germans TríasiPujol University Hospital, Badalona, Spain

${ }^{\mathrm{i}}$ Rheumatology Department, Complexo Hospitalario Universitario de Vigo, Spain

${ }^{\mathrm{j}}$ Rheumatology Department, Navarra Hospital, Navarra, Pamplona, Spain

${ }^{\mathrm{k}}$ Rheumatology Department, Príncipe de Asturias University Hospital, Madrid, Spain

${ }^{1}$ Rheumatology Department, Salamanca Clinic University Hospital, Salamanca, Spain

${ }^{\mathrm{m}}$ Rheumatology Department, Complexo Hospitalario Universitario de Ourense, Ourense, Spain

${ }^{\mathrm{n}}$ Rheumatology Department, León Hospital, León, Spain

${ }^{\circ}$ Rheumatology Department, Marqués de Valdecilla University Hospital, Santander, Spain

${ }^{\mathrm{P}}$ Rheumatology Department, Puertadel Hierro-Majadahonda Hospital, Madrid, Spain

${ }^{\mathrm{q}}$ Rheumatology Department Basurto Hospital, Basurto, Spain

r José Ángel, Rheumatology Department, Hospital Insular of Gran Canaria, Las Palmas de Gran Canaria, Spain

${ }^{\mathrm{s}}$ RheumatologyDepartment, Tenerife Clinic Hospital, Tenerife, Spain

${ }^{\mathrm{t}}$ Rheumatology Department, Virgen Macarena Hospital, Sevilla, Spain

${ }^{u}$ RheumatologyDepartment, Miguel Servet University Hospital, Zaragoza, Spain

${ }^{\mathrm{v}}$ RheumatologyDepartment, Virgen de laArrixaca University Hospital, Murcia, Spain

${ }^{\mathrm{w}}$ RheumatologyDepartment, La Paz University Hospital, Madrid, Spain

${ }^{\mathrm{x}}$ Rheumatology Department, Complexo Hospitalario Universitario de Vigo, IRIDIS (Investigation in Rheumatology and Immune-Mediated Diseases) Group Instituto de Investigación Sanitaria Galicia Sur (IISGS), Vigo, Spain
}

\footnotetext{
* Corresponding author.

E-mail addresses: vtorrente@hsjdbcn.org.

vicente.torrentesegarra@sanitatintegral.org (V. Torrente-Segarra), iruafer@gobiernodecanarias.org (I. Rúa-Figueroa), mgalindo@h12o.es (M. Galindo-Izquierdo), jaime.calvo@scsalud.es (J. Calvo-Alén), aolive.germanstrias@gencat.cat (A. Olivé-Marqués),

coral.mourino.rodriguez@sergas.es (C. Mouriño-Rodríguez),eva.salgado@sergas.es

(E. Salgado), rblanco@humv.es (R. Blanco), jlandreu@arrakis.es (J.L. Andreu),

OLAIABEGONA.FERNANDEZBERRIZBEITIA@osakidetza.net (O. Fernández-Berrizbeitia),

apecondon@ono.com (A. Pecondón-Español),gemabonilla@ser.es (G. Bonilla),

jose.maria.pego.reigosa@sergas.es (J.M. Pego-Reigosa).
} 


\section{A R T I C L E I N F O}

Keywords:

Juvenile Systemic Lupus Erythematosus

Organ damage, Mortality

Cluster analysis

RELESSER

\begin{abstract}
A B S T R A C T
Objectives: To identify patterns (clusters) of damage manifestation within a large cohort of juvenile SLE (jSLE) patients and evaluate their possible association with mortality.

Methods: This is a multicentre, descriptive, cross-sectional study of a cohort of 345 jSLE patients from the Spanish Society of Rheumatology Lupus Registry. Organ damage was ascertained using the Systemic Lupus International Collaborating Clinics Damage Index. Using cluster analysis, groups of patients with similar patterns of damage manifestation were identified and compared.

Results: Mean age (years) \pm S.D. at diagnosis was $14.2 \pm 2.89 ; 88.7 \%$ were female and $93.4 \%$ were Caucasian. Mean SLICC/ACR DI \pm S.D. was $1.27 \pm 1.63$. A total of $12(3.5 \%)$ patients died. Three damage clusters were identified: Cluster 1 (72.7\% of patients) presented a lower number of individuals with damage (22.3\% vs. $100 \%$ in Clusters 2 and 3, $P<0.001)$; Cluster 2 (14.5\% of patients) was characterized by renal damage in $60 \%$ of patients, significantly more than Clusters 1 and $3(P<0.001)$, in addition to increased more ocular, cardiovascular and gonadal damage; Cluster 3 (12.7\%) was the only group with musculoskeletal damage (100\%), significantly higher than in Clusters 1 and $2(P<0.001)$. The overall mortality rate in Cluster 2 was 2.2 times higher than that in Cluster 3 and 5 times higher than that in Cluster $1(P<0.017$ for both comparisons).

Conclusions: In a large cohort of jSLE patients, renal and musculoskeletal damage manifestations were the two dominant forms of damage by which patients were sorted into clinically meaningful clusters. We found two clusters of jSLE with important clinical damage that were associated with higher rates of mortality, especially for the cluster of patients with predominant renal damage. Physicians should be particularly vigilant to the early prevention of damage in this subset of jSLE patients with kidney involvement.
\end{abstract}

(C) 2018 Elsevier Inc. All rights reserved.

\section{Introduction}

Systemic lupus erythematosus (SLE) is a complex systemic rheumatic disease in which several domains should be assessed: disease activity, organ damage and health-related quality of life [1]. As survival in SLE patients has improved over the past decades [2,3], evaluation of organ damage has become more relevant.

Damage in SLE is defined as an irreversible change, unrelated to active inflammation, that occurs after diagnosis of the disease, and which is present for at least 6 months [4]. The Systemic Lupus International Collaborating Clinics (SLICC)/American College of Rheumatology (ACR) Damage Index (SDI) has been shown to be a valid and reliable measure for damage in SLE [4-6-].

Several studies have shown that organ damage predicts future mortality in SLE patients, usually by using the global SDI score to analyze the association between damage and mortality [7-15-]. Very few studies have assessed damage clustering and the impact of the different SDI domains on survival [16-18]. Recently, our RELESSER Study Group has detected three clusters associated with different mortality rates in our entire SLE cohort, mainly based on the proportion of patients with musculoskeletal and cardiovascular damage [18]. Our group also compared juvenile-onset SLE (jSLE) and adultonset SLE (aSLE) patients within RELESSER without finding significant differences in the mortality rate between the two subgroups [19]. However, cluster analysis of damage manifestations and their potential association with mortality in jSLE patients has not been carried out.

Therefore, the primary objective of our study was to identify patterns (clusters) of damage manifestation within a large cohort of jSLE patients. In addition, we sought to evaluate the potential association of these clusters with the risk of mortality in jSLE.

\section{Patients and methods}

\section{Research study network}

The Registry of Systemic Lupus Erythematosus Patients of the Spanish Society of Rheumatology (RELESSER) is a hospital-based registry that consists of two stages. The first one is a cross-sectional stage whose main objective is to describe the characteristics and comorbidities of the patients diagnosed with SLE in Spain. This is followed by a longitudinal follow-up stage over time with repeated yearly visits. The RELESSER Registry was conducted by the Systemic Autoimmune Diseases Study Group of the Spanish Society of Rheumatology and involved 45 rheumatology departments. All investigators signed a written commitment before participating. Informed consent was obtained from all patients who participated in the longitudinal stage. The study was approved by the local ethics committees of the participating centres in accordance with the Declaration of Helsinki's guidelines for research in humans.

\section{Study design}

This is a national, multicentre, descriptive study of a cohort with a cross-sectional analysis, conducted at the time of the last medical visit, of every patient (or death, if applicable). A detailed description of its methodology has been previously published [18]. Briefly, a specific protocol was created to collect $\sim 400$ variables per patient. Information was obtained by reviewing clinical histories and electronically compiled. Before starting the data collection, in order to minimize missing data, all investigators were encouraged to carry out a census of their SLE patients and complete any missing data. In order to ensure data homogeneity and quality, every item in the protocol had a highly standardized definition. A training course for investigators was first carried out to avoid information bias and all investigators had online access to guidelines on how to complete the protocol. The first patient was entered in October 2011. Electronic data collection finished in August 2012. Subsequently, a professional monitor with experience in rheumatologic studies reviewed the database and detected missing or inconsistent data, which were discussed with the principal investigators and sent to the subinvestigators for additions and corrections.

\section{Patients}

The RELESSER database includes unselected consecutive SLE patients. For the purpose of the present study, we selected all patients who fulfilled the following inclusion criteria: (a) age at SLE diagnosis $<18$ years and $(\mathrm{b}) \geq 4$ American College of Rheumatology (ACR) 1997 criteria for the classification of SLE $[19,20]$. There were no specific exclusion criteria.

In order to avoid selection bias, patients were widely and homogeneously spread across Spain. Virtually all patients with SLE treated 
Table 1a.

Demographics of patients in RELESSER with juvenile onset systemic lupus erythematosus and of every cluster $(n=345)$

\begin{tabular}{|c|c|c|c|c|c|}
\hline Variable & $\begin{array}{l}\text { All patients } \\
\quad n=345\end{array}$ & $\begin{array}{c}\text { Cluster 1 } \\
n=251(72.7 \%)\end{array}$ & $\begin{array}{c}\text { Cluster } 2 \\
n=50(14.6 \%)\end{array}$ & $\begin{array}{c}\text { Cluster } 3 \\
n=44(12.7 \%)\end{array}$ & $p$ value \\
\hline Gender, $n(\%)$ & & & & & 0.121 \\
\hline Male & $39(11.3)$ & $31(12.4)$ & $7(14.0)$ & $1(2.3)$ & \\
\hline Female & $306(88.7)$ & $220(87.6)$ & $43(86.0)$ & $43(97.7)$ & \\
\hline Ethnicity, $n(\%)$ & & & & & 0.478 \\
\hline 0 Caucasian & $313(93.4)$ & & & & \\
\hline 1 Aphrican-American & $1(0.3)$ & & & & \\
\hline 2 Latin-American & $15(4.5)$ & & & & \\
\hline 3 Asian & $2(0.6)$ & & & & \\
\hline 4 Other & $4(1.2)$ & & & & \\
\hline Caucasian & $313(90.7)$ & $226(92.6)$ & $46(93.9)$ & $41(97.6)$ & \\
\hline Non-Caucasian & $22(6.4)$ & $18(7.4)$ & $3(6.1)$ & $1(2.4)$ & \\
\hline Age at diagnosis (years), mean \pm S.D. & $14.25 \pm 2.89$ & $14.34 \pm 2.97$ & $13.65 \pm 3.06$ & $14.45 \pm 2.17$ & 0.250 \\
\hline Age at end of follow-up (years), mean \pm S.D. & $30.14 \pm 9.35$ & $28.61 \pm 8.78^{\mathrm{a}, \mathrm{b}}$ & $32.35 \pm 8.15^{b, c}$ & $36.41 \pm 10.77^{\mathrm{a}, \mathrm{c}}$ & $<0.001$ \\
\hline Follow-up time (months), mean \pm S.D. & $208.67 \pm 112.86$ & $188.39 \pm 108.60^{\mathrm{a}, \mathrm{b}}$ & $248.76 \pm 91.63^{c}$ & $278.80 \pm 120.22^{c}$ & $<0.001$ \\
\hline
\end{tabular}

Abbreviations: Systemic Lupus International Collaboration Clinics American College of Rheumatology/Damage Index (SDI).

a Significantly different from cluster 2 .

b Significantly different from cluster 3 .

c Significantly different from cluster 1 .

in our country are referred to hospitals, thus avoiding the possibility of centre selection bias.

\section{Variables and definitions}

Around 400 variables per patient were collected in the RELESSER Registry [18]. For this study, the following variables were included in the analyses:

- Demographic features: age, gender and ethnicity.

- Chronologic data: time of first symptom of the disease, time of diagnosis of SLE, time of follow-up, date of every damage event and date of death (if applicable).

- Cumulative manifestations of damage, using the definitions of the SDI, at the time of the patient's last medical visit (or death, if applicable).

\section{Statistical analysis}

Cluster analysis was carried out by applying $k$-means statistical analysis (partitioning method) to identify those groups of SLE patients with similar patterns of damage manifestation by the end of the follow-up. The method starts with $k$ clusters (fixed a priori) and then moves patients among clusters with the goal of minimizing variability within clusters and maximizing variability between clusters. Euclidean distance was used as a measure of similarity between the damage profiles of two patients.

We ran the $k$-means analysis with 2, 3, 4 and 5 clusters and the outputs were compared to each other. A plot of the total withingroups sums of squares against the number of clusters was used to choose the optimal number of clusters.

Three clusters of patients were finally compared. The comparison of mortality among clusters was performed for the whole cohort and for the subgroup of patients with disease duration $<5$ years. Results were expressed as mean (SD) for continuous variables, and as number of patients (percentages) for binary and categorical variables. The ANOVA test was used to compare continuous variables. The chisquared test was employed to compare the frequencies of categorical variables among the different clusters. Statistical significance was concluded when $P<0.05$.

All analyses were performed with R Statistical Software, version 3.1.1 (R Foundation for Statistical Computing, Vienna, Austria).

\section{Results}

We collected data from 345 jSLE patients who fulfilled the inclusion criteria. Baseline demographics, mortality data and specific damage domain involvement from these jSLE patients are summarized in Table 1a.

\section{Cluster analysis}

Among the 345 jSLE patients, three subgroups with similar damage patterns were identified by $k$-means cluster analysis and then compared. Cluster 1 included 251 (72.7\%) patients, Cluster 250 (14.6\%) patients and Cluster $344(12.7 \%)$ patients. Characteristics within the clusters and $P$-values for between-cluster comparisons are shown in Table $1 \mathrm{~b}$.

Cluster 1 was the largest group, with mild overall damage or no damage. Patients in this cluster had both lower mean SDI scores and a lower mean number of domains involved compared to those in the other two clusters.

Cluster 2 was the smallest group, with renal damage being predominant (60\% of the patients). Ocular and cardiovascular damage were present in $54 \%$ and $20 \%$ of the cases, respectively. Musculoskeletal damage was hardly present (2\%). Patients in Cluster 2 had both the highest mean SDI score and the highest mean number of domains with damage.

All patients in Cluster 3 had musculoskeletal damage and it was most frequently associated with peripheral vascular damage (15.9\%). However, renal damage was present only in $9.1 \%$ of cases. As was similarly observed in Cluster 2, and in contrast to Cluster 1, all patients showed at least one SDI domain involvement. There was no significant difference in disease duration in Cluster 3 patients compared with those in Cluster 2.

There were a total of 12 deaths. Patients in Cluster 2 had the highest mortality (10\%), which was significantly higher than in Clusters 1 (2\%) and 3 (4.5\%), with $P=0.017$ for all comparisons. In the group of patients with disease duration $<5$ years, there were only 3 deaths so no meaningful conclusions can be drawn (Table 2).

\section{Discussion}

In a large national jSLE cohort we observed several clinical patterns of damage manifestation and their association with different risks of mortality. To the best of our knowledge, this is the first study to perform such an analysis in jSLE. We found significant differences among clusters not only in the proportion of patients with some kind 
Table 1b.

Damage, damage distribution (per SDI domain) and mortality of patients in RELESSER with juvenile onset systemic lupus erythematosus and of every cluster ( $n=345$ )

\begin{tabular}{|c|c|c|c|c|c|}
\hline Variable & $\begin{array}{l}\text { All patients } \\
\quad n=345\end{array}$ & $\begin{array}{c}\text { Cluster 1 } \\
n=251(72.7 \%)\end{array}$ & $\begin{array}{c}\text { Cluster } 2 \\
n=50(14.6 \%)\end{array}$ & $\begin{array}{c}\text { Cluster } 3 \\
n=44(12.7 \%)\end{array}$ & $\mathrm{p}$ value \\
\hline \multicolumn{6}{|l|}{ SLICC ACR/DI Damage, $n$ (\%) } \\
\hline Ocular & $32(90.3)$ & $0^{\mathrm{a}, \mathrm{b}}$ & $27(54.0)^{\mathrm{b}, \mathrm{c}}$ & $5(11.4)^{a, c}$ & $<0.001$ \\
\hline Neuropsychiatric & $38(11.0)$ & $23(9.2)$ & $7(14.0)$ & $8(18.2)$ & 0.162 \\
\hline Renal & $34(9.9)$ & $0^{\mathrm{a}, \mathrm{b}}$ & $30(60.0)^{b, c}$ & $4(9.1)^{a, c}$ & $<0.001$ \\
\hline Pulmonary & $6(1.7)$ & $2(0.8)^{b}$ & $1(2.0)$ & $3(6.8)^{c}$ & 0.019 \\
\hline Cardiovascular & $24(7.0)$ & $10(4.0)^{\mathrm{a}}$ & $10(20.0)^{c}$ & $4(9.1)$ & $<0.001$ \\
\hline Peripheral vascular & $24(7.0)$ & $12(4.8)^{b}$ & $5(10.0)$ & $7(15.9)^{c}$ & 0.018 \\
\hline Gastrointestinal & $9(2.6)$ & $7(2.8)$ & $1(2.0)$ & $1(2.3)$ & 0.940 \\
\hline Musculoskeletal & $45(13.0)$ & $0^{\mathrm{b}}$ & $1(2.0)^{b}$ & $44(100)^{a, c}$ & $<0.001$ \\
\hline Cutaneous & $9(2.6)$ & $3(1.2)^{b}$ & $3(6.0)$ & $3(6.8)^{c}$ & 0.026 \\
\hline Diabetes & $2(0.6)$ & $2(0.8)$ & 0 & 0 & 0.686 \\
\hline Premature Gonadal Failure & $17(4.9)$ & $7(2.8)^{\mathrm{a}}$ & $7(14.0)^{\mathrm{c}}$ & $3(6.8)$ & 0.003 \\
\hline Malignancy & $8(2.3)$ & $6(2.4)$ & $1(2.0)$ & $1(2.3)$ & 0.986 \\
\hline SDI score, (mean \pm S.D. & $1.27 \pm 1.63$ & $0.70 \pm 1.18^{\mathrm{a}, \mathrm{b}}$ & $2.90 \pm 1.54^{c}$ & $2.66 \pm 1.87^{c}$ & $<0.001$ \\
\hline Any damage domain involved, n (\%) & $150(43.5)$ & $56(22.3)$ & $50(100)$ & $44(100)$ & $<0.001$ \\
\hline Number of domains involved, mean \pm S.D. & $0.72 \pm 1.02$ & $0.29 \pm 0.59$ & $1.86 \pm 0.93^{c}$ & $1.89 \pm 1.18^{\mathrm{c}}$ & $<0.001$ \\
\hline Death, $n(\%)$ & $12(3.5)$ & $5(2.0)$ & $5(10.0)$ & $2(4.5)$ & 0.017 \\
\hline
\end{tabular}

Abbreviations: Systemic Lupus International Collaboration Clinics American College of Rheumatology/Damage Index (SDI).

a Significantly different from cluster 2 .

b Significantly different from cluster 3.

c Significantly different from cluster 1 .

Table 2.

Cluster analysis: damage involvement and mortality for those patients with disease duration shorter than 5 years $(n=80)$

\begin{tabular}{lcccc}
\hline & $\begin{array}{c}\text { Cluster 1 } \\
n=72(90.0 \%)\end{array}$ & $\begin{array}{c}\text { Cluster 2 } \\
n=3(3.8 \%)\end{array}$ & $\begin{array}{c}\text { Cluster 3 } \\
n=5(6.3 \%)\end{array}$ & p-values \\
\hline $\begin{array}{l}\text { Age at diagnosis (years), } \\
\text { mean } \pm \text { S.D. }\end{array}$ & $14.22 \pm 3.07$ & $13.97 \pm 3.53$ & $14.26 \pm 2.77$ & 0.949 \\
$\begin{array}{lccc}\text { Disease duration (months) } \\
\text { SDI score, mean } \pm \text { S.D. }\end{array}$ & $6.07(15,95)$ & $2.33(1.94)$ & $5.84(4.22)$ & 0.587 \\
$\begin{array}{l}\text { Patients with damage, } n(\%) \\
\text { Number of SDI domains }\end{array}$ & $0.64 \pm 1.27$ & $1.67 \pm 1.56^{\mathrm{b}}$ & $2.60 \pm 2.07^{\mathrm{a}}$ & 0.001 \\
$\quad$ involved, mean \pm S.D. & $0.17 \pm 0.41^{\mathrm{a}}$ & $1.33 \pm 0.58^{\mathrm{b}, \mathrm{c}}$ & $1.60 \pm 0.89^{\mathrm{a}}$ & $<0.001$ \\
Death, $n(\%)$ & $2(2.8)$ & $0(0)$ & $1(20.0)$ & 0.295 \\
\hline
\end{tabular}

Abbreviations: Systemic Lupus International Collaboration Clinics American College of Rheumatology/Damage Index (SDI).

a Significantly different from cluster 2 .

b Significantly different from cluster 3.

c Significantly different from cluster 1.

of damage, but also in the level of damage, both in terms of the number of SDI domains affected and in the mean SDI score.

Similarly, as we previously observed in the global SLE population [18], three distinct clusters based on damage manifestations were identified, which we then explored for their association with mortality. Cluster 1 was the largest and the only one to include patients without damage. All patients in Clusters 2 and 3 presented damage. However, these two clusters differed significantly in terms of the predominant damage that was present: patients with renal and cardiovascular damage mainly represented Cluster 2, while those with musculoskeletal damage comprised almost all of Cluster 3. In an interesting recent publication, intense immunosuppressant treatment and arthritis involvement were linked to a higher number of flares per year and increased mortality. However, no cluster was associated with renal involvement, probably due to the low number of patients with this manifestation (13.6\%) [19]. This observation seems very unusual in a 366 cohort of SLE patients, and no information about jSLE patients was mentioned. We previously found that jSLE patients showed a higher prevalence of renal disease than aSLE, which might easily lead to the presence of a specific cluster with a clear predominance of renal damage. The latter differed from what we observed in aSLE patients, where a cluster with predominant cardiovascular damage associated with higher mortality was found $[18,20]$.

Predictors of damage in 473 jSLE patients followed into adulthood (mean follow-up time 5.63years) have been recently assessed [21]. The authors found the following factors associated to damage through time: baseline life-threatening disease involvement, AfroCaribbean ethnicity, diagnosis within 1980s decade, each $10-\mathrm{mg}$ increment of prednisone, cyclophosphamide use and some of the SLEDAI-2K items (psychosis, visual changes, lupus headache, pleurisy, proteinuria, haematuria and fever). Antimalarial use protected against increase of damage. In this study, a $2 \%$ of mortality rate was found, approximately half of ours, but no associated factors were evaluated,

In our study, mortality was higher in Cluster 2 compared to the other clusters. This finding could be explained by the fact that cardiovascular involvement was more frequent in Cluster 2 (present in 20\% of cases) and also associated with renal damage (present in $60 \%$ of cases). Renal damage has been described as a prognostic factor associated with higher mortality in SLE [17]. Based on our results, rheumatologists and pediatric rheumatologists should be aware of the presence of a specific subgroup of patients who mainly present renal damage, but who may also present cardiovascular damage and high mortality rates in jSLE. Whether this mortality might be prevented or lowered is still unknown, but we believe better disease management might prevent future renal failure, diminish renal damage, and probably mortality. It might also prove interesting to more carefully assess cardiovascular disease in this specific patient subgroup. Based on previous research in aSLE, the LUMINA cohort and the RELESSER registry revealed an association between mortality and renal and cardiovascular damage, respectively $[17,18]$. These observations were confirmed in our study in jSLE as Cluster 2 combined renal and cardiovascular damage and mortality. Since more jSLE patients suffer from renal disease than adults and because it is also more severe at onset and throughout disease evolution, such observations become even more relevant for physicians who treat jSLE patients. In our previous study comparing aSLE and jSLE features, we pointed out not only that renal disease more frequently flared in jSLE compared to aSLE after its onset, but also that the histologic class changed in $25 \%$ of re-biopsied kidneys. It is imperative to properly assess renal disease in SLE in order to be even more precise and aggressive, when necessary, in treating jSLE patients [20].

The limitations of this study include its cross-sectional design, since longitudinal studies can more adequately assess mortality over time. The low number of deaths within the jSLE group could also have limited our analyses. Another limitation is that the SDI index was not specifically designed for assessing damage in jSLE (for instance, the possibility of curing osteonecrosis in children, or the 
potential presence of limited growth, which is not included in SDI). On the other hand, our study has several strengths: the rigorous manner in which clinical data were collected to ensure comprehensiveness and the large number of patients derived from numerous centres across the entire country with a long follow-up duration and an appreciable number of deaths over time.

\section{Conclusions}

In conclusion, this is the first study to analyze and identify different patterns (clusters) of damage manifestations within a large cohort of jSLE patients. Renal and musculoskeletal damage were the two dominant forms of damage used to sort these patients into clinically meaningful clusters. Our study suggests that musculoskeletal, and particularly renal damage, manifestations are significantly associated with an increased risk of mortality. Therefore, physicians should pay special attention to the early prevention of damage in this particular subset of jSLE patients who present kidney involvement.

\section{Disclosure statement}

Dr. Pego-Reigosa is supported by grant 316265 (BIOCAPS) from the European Union 7th Framework Program (FP7/REGPOT-20122013.1). The FIS Grant PI11/02857 (Instituto Carlos III, Fondos FEDER) has supported this work.

Francisco Javier López-Longo has received speaker fees from Abbvie, Roche Farma, Bristol-Myers Squibb, Pfizer, UCB, MSD, Actelion. Francisco Javier López Longo has received research funding from Abbvie and GSK.

The RELESSER Registry was funded by grants from GSK, Roche, UCB and Novartis.

The other authors have declared no conflicts of interest.

All authors have approved the final manuscript.

\section{Supplementary material}

Supplementary data associated with this article can be found in the online version at https://doi.org/10.1016/j.semarthrit.2018.09.005.

\section{References}

[1] Strand V, Gladman D, Isenberg D, et al. Outcome measures to be used in clinical trials in systemic lupus erythematosus. J Rheumatol 1999;26:490-7.

[2] Doria A, Briani C. Lupus: improving long-term prognosis. Lupus 2008;17:166-70.

[3] Mak A, Cheung MW, Chiew HJ, Liu Y, Ho RC. Global trend of survival and damage of systemic lupus erythematosus: meta-analysis and meta-regression of observational studies from the 1950s to 2000s. Semin Arthritis Rheum 2012;41:830-9.
[4] Gladman D, Ginzler, Goldsmith E, et al. The development and initial validation of the Systemic Lupus International Collaborating Clinics/American College of Rheumatology damage index for systemic lupus erythematosus. Arthritis Rheum 1996;39:363-9.

[5] Gladman DD, Urowitz MB, Goldsmith, et al., et al. The reliability of the Systemic Lupus International Collaborating Clinics/American College of Rheumatology damage index in patients with systemic lupus erythematosus. Arthritis Rheum 1997;40:809-13.

[6] Gladman DD, Goldsmith CH, Urowitz MB, et al. The Systemic Lupus International Collaborating Clinics/American College of Rheumatology (SLICC/ACR) damage index for Systemic lupus erythematosus international comparison. J Rheumatol 2000;27:373-6.

[7] Mak A, Isenberg DA, Lau CS. Global trends, potential mechanisms and early detection of organ damage in SLE. Nat Rev Rheumatol 2013;9:301-10.

[8] Sutton EJ, Davidson JE, Bruce IN. The Systemic Lupus International Collaborating Clinics (SLICC) damage index: a systematic literature review. Semin Arthritis Rheum 2013;43:352-61.

[9] Rahman P, Gladman DD, Urowitz MB, Hallett D, Tam LS. Early damage as measured by the SLICC/ACR damage index is a predictor of mortality in systemic lupus erythematosus. Lupus 2001;10:93-6.

[10] Alarcon GS, McGwin G Jr., Bastian HM, et al. Systemic lupus erythematosus in three ethnic groups.VII [correction of VIII]. Predictors of early mortality in the LUMINA cohort. LUMINA Study Group. Arthritis Rheum 2001:45:191-202.

[11] Nived O, Jonsen A, Bengtsson AA, Bengtsson C, Sturfelt G. High predictive value of the Systemic Lupus International Collaborating Clinics/American College of Rheumatology damage index for survival in systemic lupus erythematosus. J Rheumatol 2002;29:1398-400.

[12] Cardoso CR, Signorelli FV, Papi JA, Salles GF. Initial and accrued damage as predictors of mortality in Brazilian patients with systemic lupus erythematosus: a cohort study. Lupus 2008;17:1042-8.

[13] Chambers SA, Allen E, Rahman A, Isenberg D. Damage and mortality in a group of British patients with systemic lupus erythematosus followed up for over 10 years. Rheumatology 2009;48:673-5.

[14] Bruce IN, O'Keeffe AG, Farewell V, Hanly JG, Manzi S, Su L, et al. Factors associated with damage accrual in patients with systemic lupus erythematosus: results from the Systemic Lupus International Collaborating Clinics (SLICC) inception cohort. Ann Rheum Dis 2015;74:1706-13.

[15] Yee CS, Su L, Toescu V, Hickman R, Situnayake D, Bowman S, Farewell V, et al. Birmingham SLE cohort: outcomes of a large inception cohort followed for up to 21 years. Rheumatology 2015;54:836-43.

[16] Stoll T, Seifert B, Isenberg DA. SLICC/ACR damage index is valid, and renal and pulmonary organ scores are predictors of severe outcome in patients with systemic lupus erythematosus. Br J Rheumatol 1996;35:248-54.

[17] Danila MI, Pons-Estel GJ, Zhang J, Vilá LM, Reveille JD, Alarcón GS. Renal damage is the most important predictor of mortality within the damage index: data from LUMINA LXIV, a multiethnic US cohort. Rheumatology 2009;48:542-5.

[18] Pego-Reigosa JM, Lois-Iglesias A, Rúa-Figueroa Í, Galindo M, Calvo-Alén J, de UñaÁlvarez J, et al. Relationship between damage clustering and mortality in systemic lupus erythematosus in early and late stages of the disease: cluster analyses in a large cohort from the Spanish Society of Rheumatology Lupus Registry. Rheumatology 2016;55:1243-50.

[19] De Marchi G, Quartuccio L, Zuliani F, Bond M, De Vita S. The relevance of cluster analyses to stratify systemic lupus erythematosus: increased mortality with heavier treatment. Clin Exp Rheumatol 2017;35:873-4.

[20] Torrente-Segarra V, Salman Monte TC, Rúa-Figueroa I, Sánchez-Alonso F, LópezLongo FJ, Galindo-Izquierdo M, et al. RELESSER study group of the Spanish society of rheumatology (SER) and the study group of systemic autoimmune diseases of the SER (EAS-SER). Juvenile- and adult-onset systemic lupus erythematosus: a comparative study in a large cohort from the Spanish Society of Rheumatology Lupus Registry (RELESSER). Clin Exp Rheumatol 2017;35:1047-55.

[21] Lim LSH, Pullenayegum E, Lim L, Gladman D, Feldman B, Silverman E. From childhood to adulthood: the trajectory of damage in patients with juvenile-onset systemic lupus erythematosus. Arthritis Care Res 2017;69:1627-35 